\title{
Sickness and Abnormal Behaviors as Indicators of Animal Suffering
}

\section{Michele Panzera}

Laboratory of Veterinary Comparative Ethology, Department of Veterinary Science, University of Messina, Italy

doi: 10.7358/rela-2013-001-panz

mpanzera@unime.it

\begin{abstract}
The welfare status of an animal represents the integrated outcome of all sensory and other neural inputs from within its body and from the environment. These inputs are processed and interpreted by the animal's brain according to its species-specific and individual nature and experiences, and then are perceived consciously. That being said, for an animal to be able to perceive states that we believe would reflect its welfare, the animal must be alive and conscious, and it must also be sentient. Previous studies, which I cite and describe further later in this paper, show that the brains of animals, especially mammals, have enough complexity to process mental states. The mental abilities of an animal arise as a result of sensory and other neural inputs linked to nutritional, environmental, bealth and behavioral components of physical or functional abilities. They are also linked to cognitive-neural inputs and activity related to external challenge. All of these components are integrated and expressed mentally as varying degrees of thirst, bunger, weakness, debility, breathlessness, nausea, sickness, pain, distress, fear, anxiety, belplessness, boredom and so on.
\end{abstract}

Keywords: Bioethics, animal welfare, sentient, feelings, behavior, allostasis, stress, depression, cytokines, sickness behavior.

\section{INTRODUCTION}

Many scientific reports have shown that even animals possess the physiological requirements to be considered 'sentient'. Possessing a mastery of sentience, from a bioethical point of view, means that animals are not only objects of moral duties but also that they are the owners of rights. Pain perception in animals, in terms of physiological mechanisms of pain detection, has been indirectly confirmed by using central and peripheral analgesics on experimental animals in pharmaceutical research. In light of these findings, cognitive ethological studies have also found that animals can translate the

Relations - 1.I - June 2013

http://www.ledonline.it/Relations/ 
central representation of pain perception not only as physical hurt but also as emotional suffering. Further experimental research has shown that emotional suffering can be generated through behavioral deprivation induced by an intensive farming system. It is clear that an animal's 'quality of life' depends on its possibility to express natural behavior. An animal's emotional suffering induces metabolic modifications that alter the organoleptic properties of animal products.

\section{THE ADAPTIVE RESPONSE OF STRESS}

Scientific analysis of animal welfare have used a wide range of indicators as proxy measures of an animal's wellbeing (Broom and Johnson 1993; Squires 2003; Webster 2005). These indicators can be divided into four categories: pathological, physiological, behavioral and productive (Smidt 1983). Classically, stress is defined as a threat to physiological and emotional homeostasis to which the organism, in order to survive, responds with a large number of adaptive responses. Stress can be either acute or chronic. Chronic stress is at times referred to as 'distress'. It has been suggested that chronic stress can act as a predisposing factor in the onset of illness, especially in humans affected by depression, and that an individual's ability or lack of ability to cope with stress may be a predisposing factor to psychiatric illness (Zacharko and Anisman 1991; Willner 1995; Leonard and Song 1996). Because of these findings, physiological indicators have been used to measure animal welfare. Examples of these indicators include cortisol and beta-endorphin levels; assessments related to the nutritional status of the animals; and pathologic changes in specific tissues (such as heart, muscle, liver, adrenal glands, etc.) (Barbieri and Nassuato 2006). Any evaluation of physiological indicators should take into consideration the age of animals, as there is evidence that the ability to experience stress and pain is different in young animals than it is in adult animals (Fitzgerald 1999; EFSA 2005). In addition, animal welfare can be measured by studying an animal's behavioral responses to stressful situations. For example, one could measure the apparent motivation of an animal to obtain access to desired objects (such as food, a companion, or bedding) or to perform some specific behavior (feeding, social interactions, etc.) (Veissier et al. 2000).

The stress response is a conserved, physiological coping reaction to adverse environmental conditions. Examples of adverse environmental conditions can include physical or psychological constraints, injuries, trauma, poor microclimate and infectious diseases. Under these circum- 
stances, immune responses such as stress and inflammation are an ancestral, overlapping set of responses aimed at neutralizing the stimuli perturbing homeostasis (Ottaviani and Franceschi 1998). The complex interaction between the immune system and the stress/inflammation complex has mainly developed through the phylogenetic evolution of vertebrates in which a redundant, diversified system of cytokines and chemokines develops. Behavioral responses to psycho-sensorial stimuli and immune responses to antigenic stimuli can be viewed as two subsystems of an integrated complex aimed to provide optimal conditions for the host's survival and adaptation. This integrated complex is based on a signalling feedback network regulated by neuroendocrine and immunological mediators. Acute and chronic stress conditions have repercussions not only on an animal's physical homeostasis, but also on its emotional homeostasis. Allostasis is an adaptive and dynamic strategy, actuated by complex living organisms to maintain a balance between their own physiological systems - involved in homeostasis process - and the external environment, in response to both predictable and unpredictable events (McEwen 2000; McEwen and Wingfield 2003).

\section{SHORT AND ACUTE STRESS}

This allostatic system is elicited by animals through different coping strategies based on the type and duration of stress stimuli. The Hypothalamus Pituitary Adrenal-axis (HPA-axis) system and Hypothalamus Pituitary Gonads-axis (HPG-axis) system are activated when an animal is under short-term stress conditions and exhibiting proactive and reactive coping strategies, and fight/flight reactions. Animals actuate these physiological and behavioral modifications during daily routines that have an energetic 'cost' for an organism such as feeding, sheltering, breeding, and migration. These well-known physiological and behavioral responses are regulated by the sympathetic branch of the autonomic nervous system. Simultaneously, activation of the HPA-axis induces the release of Corticotrophin Realising Factor (CRF) by the hypothalamus, stimulating synthesis and release of the AdrenoCorticoTropic Hormone (ACTH) which in turn stimulates the adrenal glands to activate the glucocorticoid hormones and increases levels of catecholamine (adrenalin and/or noradrenalin). Severe or long-term stress can lead to a disturbance of physiological and behavioral homeostasis and an emotional state identified as distress, which includes reduced productive or reproductive fitness, passive coping strategies and illness. Depression in humans is characterized by high levels of CRF which causes 
HPA-axis over-activation. Hypercortisolaemia is also a common abnormality found in depression in human patients (Owens and Nemeroff 1991; Dinan 1994). In fact, in depressed subjects, there seems to be a defect in the inhibitory cortisol feedback mechanism which acts on CRF secretion, leading the organism to maintain elevated cortisol levels and reduced immunological activity (Dinan 1994). Moreover, cytokines and prostaglandins mediate sickness behaviors, such as lethargy, anorexia, curtailment of social and reproductive activities (Hart 1988). They have also been associated with a concurrent decrease in learning and in memory - both of which are maladaptive responses towards an unsuitable environment (Dunn and Swiergiel 1998; Dantzer 2001). Cytokines are secreted in response to both infectious and non-infectious stimuli, such as experimental stress conditions in rodents has shown (Lemay, Vander, and Kluger 1990; Zhou et al. 1993; Shintani et al. 1995; Deak et al. 1997).

Friedman et al. demonstrated the capacity of cytokines to enter the Central Nervous System (CNS) after an increase in the permeability of the Blood Brain Barrier (BBB) in mice subjected to an acute swim stress (Friedman et al. 1996). Additional studies have attempted to evaluate and characterize the controversial role of BBB permeability under stress conditions (Minami et al. 1991; Goujon et al. 1995; Plotkin et al. 2000; Ovadia et al. 2001).

Currently, it is postulated that cytokines produced in the periphery organs act predominantly on the circumventricular organs within the brain, particularly via the organum vasculosum of the laminae terminalis (OVLT) (Hopkins and Rothwell 1995). BBB permeability is not as dense in the circumventricular organs as it is in other parts of the brain. At the OVLT, cytokines are believed to bind to glial cells, which in turn produce cytokines and other mediators such as prostaglandins, particularly $\mathrm{PGE}_{2}$. Other studies have shown the presence of an active transport mechanism that could be the way of entry through the $\mathrm{BBB}$ when plasma concentrations of cytokines are very high (Banks, Kastin, and Durham 1989; Banks et al. 1991).

Cytokines and their receptors have been identified in many tissues, including in the peripheral and central nervous system (Schobitz, Holsboer, and Ron de Kloet 1994). Histochemical studies using rodent and human tissues have revealed that IL-1, IL-6 and TNF-alpha are expressed in neurons and glial cells within the CNS under non-inflammatory conditions, albeit in small quantities (Schobitz, Holsboer, and Ron de Kloet 1994).

Thanks to their general properties of pleiotropism, redundancy, synergism and antagonism, cytokines have an integrated and interdependent 
function in the immunological system. They operate within a complex network and may act either synergistically or antagonistically, thus influencing the production of cytokines from other cell types and inducing a consequent neuroendocrine and immune response associated with behavioral changes. However, the exact relationship between cytokines and characteristics of depression remain to be identified. Elevated cytokines levels (e.g. IL-1, IL-6, TNF-alpha, IFN-gamma) contribute to some aspects of the abnormal behaviors in animals and atypical symptomatology, including increase in sleeping, muscle fatigue, and decrease of eating (Dantzer 1991; Dantzer 2001; Anisman and Merali, 2002). Altered cytokine activity seen in animal and/or human depression may actually be caused by increased stressor perception (or actual experience) and is believed to be based specifically on the duration of the stress. Situations which cause discomfort, such as unsuitable environment or lifestyle conditions, lead to a reduced perception of uplifting events and increased feelings of loneliness with consequent physiological and emotional alterations, where cytokines have an important role as biomodulators (Ravindran et al. 1995; 1999).

\section{StRess AND ANIMAL WELFARE}

Animals kept in laboratories and/or intensive housing systems are exposed to different stress situations and often do not have the possibility to avoid aversive stimuli by showing specific adaptive reactions. Broom stated that welfare is poor when the individual has difficulty in coping with its environment (Broom 1991). When the environment also causes a reduced fitness level, an animal may fail to cope with stress, and abnormal sickness behaviors may occur (Wiepkema 1987; Dantzer 1991; Cronin, Wiepkema, and Hofstede 1984; Wechsler 1995; McEwen 2000; McEwen and Wingfield 2003).

The protection and the welfare requirements for animals is an area covered by a wide range of EU legislation (these protections are created most commonly in the interest of guaranteeing the quality of animal products such as meat, eggs, and milk). Consumers are sensitized to animal welfare campaigns because of slogans like "The quality of food depends on health and welfare of the animal that produce it".

Because we have witnessed a demonstration of animals' negative 'capacity', such as pain and suffering, we must intuitively consider the possibility that animals also have positive 'capacity', such as intention, expectation and gratification. Both of these capacities ensure emotional homeostasis. Many studies have found that animals in intensive husbandry 
systems are unable to express a normal range of behavioral patterns. The occurrence of abnormal behavior in animals is therefore an expression of a central alteration of their emotional sphere.

To prevent poor welfare, indicative of a poor physical and emotional state, housing systems should be designed to allow animals to perform specific behavioral habits and achieve physical and behavioral homeostasis.

\section{CONCLUSION}

From an anthropocentric view, animals are often considered only for their utility for humans - but this should not always be so. Mental and physical integrity, in a holistic sense, determine an animal's capacity. It's possible to ensure this integrity through the satisfaction of all animal needs - all of which can be achieved through different animal behaviors. If animal integrity is injured then altered emotional homeostasis can eventually translate to abnormal behavior. Animal integrity is ensured when animals can live in an environment that allows them to manifest their full 'capacity', not only those capacities that are 'convenient' for humans ${ }^{1}$. In fact, by recognizing animals' positive and negative capabilities, it follows our duty to ensure them the possibility to cope with their environment in order to live a good life.

\section{REFERENCES}

Anisman, Hymie, and Zul Merali. 2002. "Cytokines, Stress, and Depressive Illness”. Brain, Behavior and Immunology 16 (5): 513-24. doi: 10.1016/S08891591(02)00009-0.

Banks, William A., Abba J. Kastin, and Debra A. Durham. 1989. "Bidirectional Transport of Interleukin-1 Alpha across the Blood Brain Barrier". Brain Research Bulletin 23 (6): 433-7. doi: 10.1016/0361-9230(89)90185-8.

Banks, William A., et al. 1991. "Human Interleukin (IL) $1 \alpha$, Murine IL-1 $\alpha$ and Murine IL-1 $\beta$ Are Transported from Blood to Brain in the Mouse by a Shared Saturable Mechanism". Journal of Pharmacology and Experimental Therapeutics 259 (3): 988-96.

Barbieri, Sara, and Caludia Nassuato, eds. 2006. Basic Information for the Development of the Animal Welfare. Risk Assessment Guidelines (EFSA/AHAW/2006/01). Parma: EFSA.

1 For convenience of humans, animals use both therapeutic and empathic capacities in different ways during pet therapy. The capacity of animals to take care of patients is a demonstration of emotional intelligence.

Relations - 1.I - June 2013

http://www.ledonline.it/Relations/ 
Broom, Donald M. 1991. "Assessing Welfare and Suffering”. Behavioural Processes 25 (2-3): 117-23. doi: 10.1016/0376-6357(91)90014-Q.

Broom, Donald M., and Kenneth G. Johnson. 1993. Stress and Animal Welfare. London: Chapman and Hall.

Cronin, Gregory M., Pieter R. Wiepkema, and Gert J. Hofstede. 1984. "The Development of Stereotypies in Tethered Sows". In Proceedings of the International Congress on Applied Ethology in Farm Animals, edited by Jürgen Unshelm, Gerrit van Putten, Klaus Zeeb, and Ingvar Eksebo, 97-100. Kiel, Germany: KTBL Darmstadt.

Dantzer, Robert. 1991. "Stress, Stereotypies and Welfare". Behavioural Processes 25 (2-3): 95-102. doi: 10.1016/0376-6357(91)90012-O.

2001. "Cytokine-induced Sickness Behavior: Where Do We Stand?". Brain, Behavior, and Immunity 15 (1): 1-18. doi: 10.1006/brbi.2000.0613.

Deak, Terrence, et al. 1997. "Evidence That Brief Stress May Induce the Acute Phase Response in Rats”. American Journal of Physiology 273 (6-2): R1998-2004.

Dinan, Timothy G. 1994. "Glucocorticoids and the Genesis of Depressive Illness. A Psychobiological Model”. The British Journal of Psychiatry 164: 365-71. doi: 10.1192/bjp.164.3.365.

Dunn, Adrian J., and Artur H. Swiergiel. 1998. "The Role of Cytokines in Infectionrelated Behavior". Annals of the New York. Academy of Sciences 840: 577-85. doi: 10.1111/j.1749-6632.1998.tb09596.x.

EFSA. 2005. "Opinion of the Scientific Panel AHAW Related to the Aspects of the Biology and Welfare of Animals Used for Experimental and Other Scientific Purpose (EFSA-Q-2004-105)". European Food Safety Agency Journal 292: 1-136.

Fitzgerald, Marie. 1999. "Development and Neurobiology of Pain". In Textbook of Pain. $4^{\text {th }}$ edition, edited by P.D. Wall and R.D. Melzack, 235-51. Edinburgh, UK: Churchill Livingstone.

Friedman, Alon, et al. 1996. "Pyridostigmine Brain Penetration under Stress Enhances Neuronal Excitability and Induces Early Immediate Transcriptional Response”. Nature Medicine 2: 1382-5. doi: 10.1038/nm1296-1382.

Goujon, E., et al. 1995. "Stress Downregulates Lipopolysaccharide-induced Expression of Proinflammatory Cytokines in the Spleen, Pituitary, and Brain of Mice". Brain, Behavior, and Immunity 9 (4): 292-303. doi: 10.1006/brbi.1995.1028.

Hart, Benjamin L. 1988. "Biological Basis of the Behaviour of Sick Animals". Neuroscience EBiobehavioral Reviews 12 (2): 123-37. doi: 10.1016/S0149-7634(88)80004-6.

Hopkins, Stephen J., and Nancy J. Rothwell. 1995. "Cytokines and the Nervous System I: Expression and Recognition”. Trends in Neurosciences 18 (2): 83-8. doi: 10.1016/0166-2236(95)93881-W.

Lemay, Lin G., Arthur J. Vander, and Matthew J. Kluger. 1990. "The Effect of Psychological Stress on Plasma Interleukin-6 Activity in Rats". Physiology \& Bebavior 47 (5): 957-61. doi: 10.1016/0031-9384(90)90024-X.

Leonard, Brian E., and Cai Song. 1996. "Stress and the Immune System in the Etiology of Anxiety and Depression". Pharmacology Biochemistry and Behavior 54 (1): 299-303 doi: 10.1016/0091-3057(95)02158-2.

Relations - 1.I - June 2013

http://www.ledonline.it/Relations/ 
McEwen, Bruce S. 2000. "Allostasis and Allostatic Load Implications for Neuropsychopharmacology”. Neuropsychopharmacology 22: 108-24. doi: 10.1016/S0893133X(99)00129-3.

McEwen, Bruce S., and John C. Wingfield. 2003. "The Concept of Allostasis in Biology and Medicine”. Hormones and Behavior 43 (1): 2-15. doi: 10.1016/S0018506X(02)00024-7.

Minami, Masabumi, et al. 1991. "Immobilization Stress Induces Interleukin-l mRNA in the Rat Hypothalamus”. Neuroscience Letters 123 (2): 254-6. doi: 10.1016/03043940(91)90944-O.

Ottaviani, Enzo, and Claudio Franceschi. 1998. "A New Theory on the Common Evolutionary Origin of Natural Immunity, Inflammation and Stress Response: the Invertebrate Phagocytic Immunocyte as an Eye-witness". Domestic Animal Endocrinology 15 (5): 291-6. doi: 10.1016/S0739-7240(98)00021-6.

Ovadia, Haim, et al. 2001. "Evaluation of the Effect of Stress on the Blood-brain Barrier: Critical Role of the Brain Perfusion Time”. Brain Research 905 (1-1): 21-5. doi: 10.1016/S0006-8993(01)02361-7.

Owens, Michael J., and Charles B. Nemeroff. 1991. "Physiology and Pharmacology of Corticotropin-releasing Factor”. Pharmacological Reviews 43 (4): 425-73.

Plotkin, Scott. R., et al. 2000. "Differential Transport of Rat and Human Interleukin-1 $\alpha$ across the Blood-brain Barrier and Blood-testis Barrier in Rats". Brain Research 881 (1): 57-61. doi: 10.1016/S0006-8993(00)02772-4.

Ravtndran, Arun V., et al. 1995. "Lymphocyte Subset in Major Depression and Dysthymia: Modification by Antidepressant Treatment". Psychosomatic Medicine 57 (6): 555-63.

Ravtndran, Arun V., et al. 1999. “Treatment of Primary Dysthymia with Group Cognitive Therapy and Pharmacotherapy: Clinical Symptoms and Functional Impairments". American Journal of Psychiatry 156 (10): 1608-17.

Schöbitz, Bernd, Florian Holsboer, and Ronald E. de Kloet. 1994. "Cytokines in the Healthy and Diseased Brain”. News in Physiological Sciences 9 (3): 138-42.

Shintani, F., et al. 1995. "Involvement of Interleukin-1 in Immobilization StressInduced Increase in Plasma Adrenocorticotropic Hormones and in Release of Hypothalamic Monoamines in Rat”. Journal of Neuroscience 15 (3-1): 1961-70.

Smidt, Diedrich, ed. 1983. Indicators Relevant to Farm animal Welfare. A Seminar in the CEC Programme of Coordination of Research on Animal Welfare, Organized by Dr. D. Smidt, and Held in Mariensee, 9-10 November 1982. Dordrecht, The Netherlands: Kluwer Academic Publishers.

Squires, James E. 2003. Applied Animal Endocrinology. Wallingford: CABI Publishing.

Veissier, Isabelle, et al. 2000. "Welfare of Livestock: How to Define and Evaluate?”. Developmental Medicine and Child Neurology 31 (205): 117-24.

Webster, John. 2005. Animal Welfare: Limping towards Eden. Oxford: Blackwell Publishing.

Wechsler, Beat. 1995. "Coping and Coping Strategies: a Behavioural View". Applied Animal Behaviour Science 43 (2):123-34. doi: 10.1016/0168-1591(95)00557-9.

Relations - 1.I - June 2013

http://www.ledonline.it/Relations/ 
Wiepkema, Pieter R. 1987. "Behavioural Aspects of Stress". In The Biology of Stress in Farm Animals: an Integrated Approach. Current Topics in Veterinary Medicine and Animal Science, edited by Pieter R. Wiepkema and Pieter W.M. van Adrichem, 113-38. The Hague, The Netherlands: Martinus Nijhoff.

Willner, Paul. 1995. "S-19-2 Pharmacology of Anhedonia". European Neuropsychopharmacology 5 (3): 214. doi: 10.1016/0924-977X(95)90243-7.

Zacharko, Robert M., and Hymie Anisman. 1991. "Stressor-induced Anhedonia in the Mesocorticolimbic System”. Neuroscience E Biobehavioral Reviews 15 (3): 391-405. doi: 10.1016/S0149-7634(05)80032-6.

Zhou, Daohong, et al. 1993. "Exposure to Physical and Psychological Stressors Elevates Plasma Interleukin 6: Relationship to the Activation of Hypothalamic-Pituitary-Adrenal Axis”. Endocrinology 133 (6): 2523-30. doi: 10.1210/ en.133.6.2523. 